\title{
Gender-specific association between unidirectional variation of cholesterol and cognitive decline: a longitudinal study of middle-aged and elderly adults
}

Huamin Liu

Southern Medical University https://orcid.org/0000-0002-2762-8012

Rui Zhou

Southern Medical University

Jiazhen Zheng

Southern Medical University

Minyi Zhang

Southern Medical University

Keyi Wu

Southern Medical University

Zhiwei Huang

Southern Medical University

Zelin Yuan

Southern Medical University

Xianbo Wu ( $\nabla$ wuxb1010@hotmail.com )

Southern Medical University

\section{Research}

Keywords: cognitive decline, cholesterol, unidirectional variation, gender-specific

Posted Date: February 19th, 2021

DOl: https://doi.org/10.21203/rs.3.rs-206645/v1

License: (c) (1) This work is licensed under a Creative Commons Attribution 4.0 International License.

Read Full License 


\section{Abstract \\ Background}

Higher fluctuation of cholesterol has been shown associated with cognitive decline. But the association between unidirectional variation of cholesterol and cognition remains unclear. In the present study, we examined the association between variation of cholesterol from normal to high level or from high to normal level and cognition.

\section{Methods}

A total of 4,915 participants (mean age, 57.7 years; $49.2 \%$ female) with normal cognition in baseline (2011) from the China Health and Retirement Longitudinal Study (CHARLS) were included. Plasma total cholesterol (TC), non-high-density lipoprotein cholesterol (NHDL-C), low-density lipoprotein cholesterol (LDL-C), and high-density lipoprotein cholesterol (HDL-C) were divided into two clusters according to cutoff in 2011 and 2015, respectively. Participants were divided into four groups: normal-normal, normalhigh, high-normal and high-high. Cognitive functions were assessed by episodic memory and mental intactness. The binary logistic regression was used to analyze the association of cholesterol variation with cognitive decline.

\section{Results}

Participants with increased cholesterol were more likely had lower incidence of cognitive decline. The fluctuations of cholesterol were more likely occurred in females. The odds ratio (OR) and $95 \%$ confidence interval $(\mathrm{Cl})$ of global cognitive decline in normal-high compared with normal-normal group of TC was $0.61(0.36-1.03)$, and significant only in females [OR and $95 \% \mathrm{Cl}: 0.50(0.26-0.97)$ ]; the risk of memory function decline in high-normal group compared with high-high group was increased both in males [OR and $95 \% \mathrm{Cl}: 1.98$ (1.02-4.05)] and females [OR and 95\% Cl: 1.61 (1.01-2.74)]. The similarly results were shown in NHDL-C groups [OR and 95\% $\mathrm{Cl}$ of global cognitive decline in normal-high group: 0.50 (0.26$0.95)$ for overall; 0.38 (0.17-0.85) for females]; but the risk of memory function decline in high-normal group only increased in females [OR and 95\% Cl: 1.64 (1.02-2.25)]. The risk of mental intactness decline in males significantly decreased in high-normal group of LDL-C compared with high-high group [OR and 95\% Cl: 0.30 (0.13-0.67)] and increased in high-high compared with normal-normal group [OR and 95\% Cl: $2.27(1.18-2.98)]$.

\section{Conclusions}

Longitudinal decreasing of TC or NHDL-C is detrimental to cognitive function, especially in females. But LDL-C is still a risk factor of cognitive decline in males. 


\section{Background}

Cholesterol is associated with cognitive impairment closely owing to its disturbance on cardiovascular and other cognition related risk factors [1]. Epidemiological cross-sectional and prospective studies have found higher total cholesterol (TC) or low-density lipoprotein cholesterol (LDL-C) was associated with worse cognitive function [2-4], while other similar studies put forward a reverse conclusion [5, 6]. The use of high-dose statins was associated with better visuospatial memory and executive ability, but some randomized placebo-controlled trials of lipid-lowering treatment found non-significant between-group differences of cognitive function $[7,8]$. In addition, the opposite association of cholesterol with cognitive function between males and females was also documented [9]. These discrepant results implied the potential limitations of using cholesterol to predict risk of cognitive impairment, and thus increased the complexity of making the prevention strategy.

The variability of cholesterol has been of increasing interest in recent years. Previous literatures have documented that visit-to-visit cholesterol variability was correlated with lower cognitive performance, in which the variability indices were calculated by intra-individual coefficient of variation (CV), variability independent of the mean (VIM) or standard deviation (SD), including downward and upward fluctuations $[10,11]$. A Chinese population-based prospective study found that intra-individual SD over two TC tests was correlated with cognitive decline in males, but not in females [12]. These findings highlight the importance of maintaining lipid stability for cognitive function. However, previous studies failed to consider whether the unidirectional increase or decrease in blood cholesterol is associated with cognitive impairment. It is also unknown whether there is gender difference in association of cholesterol's unidirectional variation with cognitive impairment.

The purpose of this study was to investigate whether longitudinal unidirectional increase or decrease in blood cholesterol, rather than bi-directional fluctuations, was associated with cognitive decline. The participants aged 45 years or older were recruited from the China Health and Retirement Longitudinal Study (CHARLS). TC, non-high-density lipoprotein cholesterol (NHDL-C), LDL-C, HDL-C and cognitive function were measured in 2011 and 2015. The association of cholesterol variation with cognitive decline will provide an updated strategy of primary prevention of cognitive impairment.

\section{Methods}

\section{Study population}

The CHARLS is a nationally representative survey conducted in 2011 which aimed to collect health related information over 45 years Chinese and their spouses, including social, economic, lifestyle, health status and function, etc. The investigation areas consist of 30 provinces, in which 450 villages/urban, 10,287 households were randomly selected by probabilities proportional to size. A total of 17,714 participants were recruited, and then the biennial follow up was carried out. There were 7,463 individuals who provided blood sample and tested for blood lipids both in 2011 and 2015. Of 7,463 participants, 333 
individuals did not complete the cognitive test in 2011 or 2015 . We excluded 77 individuals with Alzheimer's disease, brain atrophy or Parkinson's disease and 124 individuals under 45 years old in baseline. We additionally excluded 2,014 participants with cognitive impairment without dementia in baseline. Eventually, 4,915 individuals with normal baseline cognition were included in the study (Characteristics between included and excluded participants were presented in Supplemental Table 1; Flowchart of participants selection was presented in Supplemental Fig. 1). The survey was approved by the Institutional Review Board of Peking University, China (IRB00001052-11015). All subjects provided written informed consent in the baseline and follow up.

\section{Cholesterol measurement and assessment}

The blood sample was collected after an overnight fast by medically-trained staff of the local Centers for Disease Control and Prevention (CDC) at the survey site. At $4^{\circ} \mathrm{C}$ ambient temperature, a $4 \mathrm{ml}$ tube of whole blood was divided into plasma and buffy coat, which contains predominantly white blood cells, then stored in cryovials and frozen at $-20^{\circ} \mathrm{C}$. Plasma and buffy coat were transported to the Chinese CDC in Beijing within 2 weeks where they were placed in a deep freezer and stored at $-80^{\circ} \mathrm{C}$. Plasma TC, LDL-C and HDL-C were determined by enzymatic colorimetric test in Youanmen Center for Clinical Laboratory of Capital Medical University. NHDL-C was calculated by subtracting HDL-C from the TC. The high level of TC, NHDL-C, LDL-C and HDL-C was defined as $>240 \mathrm{mg} / \mathrm{dl},>190 \mathrm{mg} / \mathrm{dl},>160 \mathrm{mg} / \mathrm{dl}$ and $>50 \mathrm{mg} / \mathrm{dl}$, respectively, according Guidelines for the prevention and treatment of dyslipidemia in Chinese adults (revised in 2016) [13]. The blood testing was only carried out in 2011 and 2015. According cut-off of cholesterols, we defined high level and normal level in 2011 and 2015, respectively. The variation of TC, NHDL-C and LDL-C were classified into four groups: Normal-normal, normal levels both in 2011 and 2015; Normal-high, normal level in 2011 and high level in 2015; High-normal, high level in 2011 and normal level in 2015; High-high, high levels both in 2011 and 2015. Likewise, the variation of HDL-C was classified into Low-low, Low-normal, Normal-low, Normal-normal groups.

\section{Cognitive assessment}

In the CHARLS, cognitive function was evaluated through episodic memory and mental intactness. For episodic memory, each participant was asked to repeat as many as possible words after investigator read a list of 10 Chinese words clearly for him/her (immediate word recall); then they were asked to recall the same word list again in 5 minutes (delayed word recall). One successful word recall was coded as one point, and the episodic memory score was 20 points in total. The method of episodic memory measurement in this survey has been confirmed a good reliability and validity [14].

Numerical ability, time orientation and picture drawing were used to assess mental intactness. For numerical ability, each participant was asked to answer the question of subtracting 7 from 100, and subtracting five times serially. Time orientation included answer the date of investigation day (month, day and year), the day of the week and season of the year. For picture drawing, participants were shown a picture of two overlapping pentagons and asked to redraw it; he/she would gain one point if successful redraw the picture. Same as episodic memory, one correct answer or redraw picture was coded as one 
point, and the mental intactness score range from 0 to 11 points. The mental intactness is a wellestablished and valid measure as the Mini-Mental State Examination used to screen cognitively impaired [15].

Global cognitive score was the summation of episodic memory and mental intactness scores, ranging from 0 to 31. Cognitive impairment was defined as a score of less than 11 points according to previous studies [16]. In addition, the lowest tertile of difference in episodic memory or mental intactness score from 2011 to 2015 were considered as significant decline for cognitive dimension. The cognitive variation was thus determined by global cognitive decline, episodic memory decline or mental intactness decline in 2015.

\section{Covariate assessment}

The covariates in present study were collected by questionnaires in baseline survey, and consisted of age, sex, education, marital status, menopause in females, smoking, drinking, body mass index (BMI) and physical diseases (hypertension, diabetes, history of disability, heart problems and stroke). Education level was categorized as illiterate, part of primary school, primary or private school, middle school, high school or above. Marital status was grouped as married and living together, married but separated and single (Divorced, widowed, or never married). As for smoking, quit smoking defined as stop smoking for more than one year, otherwise classified as smoking all the time; the rest were classified as never smoking. Drinking status was also arranged as drinking all the time, former drinking and never drinking. According to Chinese standard of BMl, underweight, normal weight, overweight/obesity were defined as $\mathrm{BMl}<18.5 \mathrm{~kg} / \mathrm{m} 2,18.5-23.9 \mathrm{~kg} / \mathrm{m} 2$ and $\geq 24.0 \mathrm{~kg} / \mathrm{m} 2$, respectively. Physical diseases were based on self report of "Whether a doctor ever told you that you had hypertension, diabetes, history of disability, heart problems or stroke?". In addition, the systolic pressure $\geq 140 \mathrm{mmHg}$ or diastolic pressure $\geq 90$ $\mathrm{mmHg}$ at baseline measurement was also considered as hypertension; the fasting blood glucose $\geq 126$ $\mathrm{mg} / \mathrm{dl}$ or glycosylated hemoglobin $\geq 6.5 \%$ was also considered as diabetes.

\section{Statistical analyses}

The differences between participants with persistent cognitive normal and cognitive impairment of unordered categorical variables were identified using $\chi^{2}$ test, and continuous variables were Student $t$ test. The Nonparametric test of Kruskal-Wallis was used for identifying difference of ordinal categorical variables between groups. Among four groups of cholesterol, Analysis of Variance was used for continuous variables and $\chi^{2}$ test was used for categorical variables test. The post hoc Bonferroni method was applied to account for multiple comparisons, in which the normal-normal group was considering reference.

Binary logistic regression was used to evaluate the association of cholesterol variation with cognitive decline due to lack of time scale of incidence. In present study, to explore the unidirectional variation between different scale of cholesterol, we arranged the reference group: participants with normal cholesterol level in 2011 were considered as an ensemble, and the normal-normal group was reference; 
the odds ratio (OR) and 95\% confidence interval $(\mathrm{Cl})$ for normal-high group was calculated; Participants with high cholesterol level in 2011 were considered as an ensemble, and the high-high group was reference; the OR $(95 \% \mathrm{Cl})$ for high-normal group was calculated. We also calculated the OR $(95 \% \mathrm{Cl})$ for high-high group compared with normal-normal group, to verifying the risk of cognitive decline in participants with persistent high or normal cholesterol. In our logistic regression results, we presented adjusted ORs and $95 \%$ Cls. Baseline age, sex, education, marital status, smoking, drinking, BMI, physical diseases and two years average cholesterol level were adjusted. Given the inverse association of lipids with cognitive decline in the male and female reported by previous studies, we conducted the subgroup analyses categorized by gender. The menstrual status was adjusted in the female subgroup additionally.

All statistical tests were 2-sided, and the significance level was $P<0.05$. Statistical analyses were performed using SAS software, version 9.4 (SAS Institute Inc, Cary, NC).

\section{Results}

\section{Characteristics of participants}

Of 4,915 cognitive normal participants in 2011, 979 (19.91\%) occurred global cognitive decline in 2015. The mean and SD of age was $57.7 \pm 8.2$ and $49.2 \%$ participants were females. Participants with incidence of global cognitive decline were older, and females were prone to global cognitive decline than males. The female with menopause was also more likely to experience global cognitive decline. BMI was lower in participants with global cognitive decline, and fewer individuals with overweight or obesity were found in global cognitive decline group than cognitive normal group. Among individuals with global cognitive decline, the proportion of single or low education was higher. There were fewer drinker and more disabled person in global cognitive decline group, but we did not found significant difference of smoking status, hypertension, diabetes, heart problems and stroke between global cognitive decline and cognitive normal participants. Participants with incidence of global cognitive decline had higher baseline HDL-C level, while the TC, NHDL-C and LDL-C in baseline were not significantly different from participants with normal cognition (Table 1). 
Table 1

Baseline characteristics of participants with or without cognitive decline from 2011 to 2015

\begin{tabular}{|c|c|c|c|c|}
\hline Characteristics & Total & $\begin{array}{l}\text { Persistent normal } \\
\text { cognition }\end{array}$ & $\begin{array}{l}\text { Cognitive } \\
\text { decline* }^{*}\end{array}$ & $P$ \\
\hline $\mathrm{n}(\%)$ & 4915 & $3936(80.1)$ & 979 (19.9) & \\
\hline Age, years ( mean $\pm S D$ ) & $57.7 \pm 8.2$ & $56.8 \pm 7.8$ & $61.3 \pm 8.9$ & $\begin{array}{l}<.001 \\
0\end{array}$ \\
\hline Female, n (\%) & $\begin{array}{l}2416 \\
(49.2)\end{array}$ & $1874(47.6)$ & $542(55.4)$ & $<.001$ \\
\hline Menopause, n (\%) & $\begin{array}{l}1676 \\
(69.4)\end{array}$ & $1231(65.7)$ & $445(82.1)$ & $\hat{L}_{0.001}$ \\
\hline $\mathrm{BMI}, \mathrm{kg} / \mathrm{m}^{2}($ mean $\pm \mathrm{SD})$ & $23.9 \pm 3.7$ & $24.1 \pm 3.7$ & $23.1 \pm 3.4$ & $\begin{array}{l}<.001 \\
\text { L }\end{array}$ \\
\hline BMI, n (\%) & & & & $\dot{C}_{0.001}$ \\
\hline Underweight & $209(4.3)$ & $138(3.5)$ & $71(7.3)$ & \\
\hline Normalweight & $\begin{array}{l}2719 \\
(55.3)\end{array}$ & $2139(54.3)$ & $580(59.2)$ & \\
\hline Overweight or Obesity & $\begin{array}{l}1987 \\
(40.4)\end{array}$ & $1659(42.2)$ & $328(33.5)$ & \\
\hline Marital status, n (\%) & & & & $<.001$ \\
\hline Married, living together & $\begin{array}{l}4324 \\
(88.0)\end{array}$ & $3530(89.7)$ & $794(81.1)$ & \\
\hline Married, separated & $179(3.6)$ & $142(3.6)$ & $37(3.8)$ & \\
\hline Single & $412(8.4)$ & $264(6.7)$ & $148(15.1)$ & \\
\hline Education, n (\%) & & & & $<.001$ \\
\hline Illiterate & $811(16.5)$ & $410(10.4)$ & $401(41.0)$ & \\
\hline Part of primary school & $878(17.9)$ & $656(16.7)$ & $222(22.7)$ & \\
\hline Primary or private school & $\begin{array}{l}1333 \\
(27.1)\end{array}$ & $1100(27.9)$ & $233(23.8)$ & \\
\hline Middle school & $\begin{array}{l}1274 \\
(25.9)\end{array}$ & $1172(29.8)$ & $102(10.4)$ & \\
\hline
\end{tabular}

* Cognitive decline was determined by global cognitive function; BMI, body mass index; TC, total cholesterol; NHDL-C, non-high-density lipoprotein cholesterol; LDL-C, low-density lipoprotein cholesterol; HDL-C, high-density lipoprotein cholesterol. 


\begin{tabular}{|c|c|c|c|c|}
\hline Characteristics & Total & $\begin{array}{l}\text { Persistent normal } \\
\text { cognition }\end{array}$ & $\begin{array}{l}\text { Cognitive } \\
\text { decline* }\end{array}$ & $P$ \\
\hline High school or above & $619(12.6)$ & $598(15.2)$ & $21(2.2)$ & \\
\hline Smoking, n (\%) & & & & 0.101 \\
\hline Never smoking & $\begin{array}{l}2879 \\
(58.6)\end{array}$ & $2281(58.0)$ & $598(61.1)$ & \\
\hline Former smoking & $331(6.7)$ & $277(7.0)$ & $54(5.5)$ & \\
\hline All the time & $\begin{array}{l}1705 \\
(34.7)\end{array}$ & $1378(35.0)$ & $327(33.4)$ & \\
\hline Drinking, n (\%) & & & & 0.001 \\
\hline Never drinking & $\begin{array}{l}3276 \\
(66.7)\end{array}$ & $2600(66.1)$ & $676(69.1)$ & \\
\hline Former drinking & $308(6.3)$ & $231(5.9)$ & $77(7.9)$ & \\
\hline All the time & $133(27.1)$ & $1105(28.1)$ & $226(23.1)$ & \\
\hline Disability, n (\%) & $668(13.6)$ & $475(12.1)$ & $193(19.7)$ & $<_{0.001}^{<}$ \\
\hline Hypertension, n (\%) & $\begin{array}{l}1849 \\
(37.6)\end{array}$ & $1456(37.0)$ & $393(40.1)$ & 0.069 \\
\hline Diabetes, n (\%) & 705 (14.3) & $567(14.4)$ & $138(14.1)$ & 0.805 \\
\hline Heart problems, n (\%) & $583(11.9)$ & $478(12.1)$ & $105(10.7)$ & 0.219 \\
\hline Stroke, n (\%) & $84(1.7)$ & $65(1.6)$ & $19(1.9)$ & 0.532 \\
\hline $\mathrm{TC}, \mathrm{mg} / \mathrm{dl}($ mean $\pm \mathrm{SD})$ & $\begin{array}{l}193.1 \pm \\
37.2\end{array}$ & $192.8 \pm 37.2$ & $194.2 \pm 37.4$ & 0.304 \\
\hline $\begin{array}{l}\text { NHDL-C, mg/dl ( mean } \pm \\
\text { SD) }\end{array}$ & $\begin{array}{l}142.9 \pm \\
37.5\end{array}$ & $142.9 \pm 37.5$ & $142.7 \pm 37.4$ & 0.842 \\
\hline LDL-C, mg/dl ( mean \pm SD $)$ & $\begin{array}{l}116.1 \pm \\
34.6\end{array}$ & $116.2 \pm 34.4$ & $115.6 \pm 35.2$ & 0.591 \\
\hline $\begin{array}{l}\mathrm{HDL}-\mathrm{C}, \mathrm{mg} / \mathrm{dl} \text { ( mean } \pm \\
\mathrm{SD} \text { ) }\end{array}$ & $50.2 \pm 15.1$ & $49.8 \pm 15.0$ & $51.5 \pm 15.3$ & 0.002 \\
\hline
\end{tabular}

\section{Population and age distribution among cholesterol variation groups}


Majority of participants kept stable low levels of cholesterols with $86.57 \%, 87.30 \%$ and $88.71 \%$ in normalnormal group of TC, NHDL-C, LDL-C and $40.96 \%$ in low-low group of HDL-C, respectively. The proportions of females were higher in normal-high, high-normal and high-high group and lower in normal-normal group of TC, NHDL-C and LDL-C than males. While the proportion of females was lower in normal-normal group of HDL-C than males (Supplemental table 2). Compared with normal-normal group by Dunnett's $t$ Tests, the female was older in high-normal and high-high group of TC, NHDL-C and LDL-C, but the male was younger in high-normal and high-high group of NHDL-C; in the four groups of HDL-C, the male in normal-normal group was older than those in low-low group, while the female's age was not significant different (Supplemental table 3).

\section{The rates of cognitive decline among cholesterol variation groups}

The differences of cognitive decline rates were not quite obvious among four cholesterol groups. But we still found lower rates of global cognitive decline in normal-high groups of TC, NHDL-C and LDL-C. The rate of global cognitive decline in normal-normal group of HDL-C was significantly higher in males, but not in females. The rate of episodic memory decline was higher in high-normal group of TC; the increased rates were also found in high-normal group of NHDL-C and LDL-C although not significant. For mental intactness decline, significant higher rates were found in high-high group of LDL-C and normal-normal group of HDL-C, and concentrated mainly on the male (Fig. 1).

\section{Association of cholesterol variation and cognitive decline}

After multivariate adjustment, the $\mathrm{OR}$ and $95 \% \mathrm{Cl}$ of global cognitive decline in the normal-high TC group were $0.61(0.36-1.03)$ taking the normal-normal group as reference; the significant decreased OR and $95 \% \mathrm{Cl}$ was shown in females $(0.50,0.26-0.97)$ but not in males $(0.89,0.38-2.10)$. For memory function decline, the high-normal TC group had an increased OR and 95\% Cl of 1.65 (1.09-2.49) compared with high-high group; the increased risks were both significant in males (OR and $95 \% \mathrm{Cl}$ : 1.98, 1.02-4.05) and females (OR and 95\% Cl: 1.61, 1.01-2.74). There was no significant association between mental intactness declined and TC variation (Fig. 2).

The OR and $95 \% \mathrm{Cl}$ of global cognitive decline was $0.50(0.26-0.95)$ and $0.38(0.17-0.85)$ in overall and females, respectively, for normal-high NHDL-C group taking normal-normal group as reference, but not significant in males. The risk of memory function decline was significantly increased in high-normal group with $\mathrm{OR}$ and $95 \% \mathrm{Cl}$ of 1.64 (1.02-2.25) in females but not significantly increased in overall and males compared with high-high group. There was also no significant association between mental intactness declined and NHDL-C variation (Fig. 3).

When categorized by LDL-C variation, we only found the significant decreased risk of mental intactness decline for high-normal group with $\mathrm{OR}$ and $95 \% \mathrm{Cl}$ of 0.30 (0.13-0.67) compared with high-high group in males; and the increased risk for high-high group with OR and $95 \% \mathrm{Cl}$ of $2.27(1.18-2.98)$ compared with 
normal-normal group in males (Fig. 4). There were not any significant associations between HDL-C variation and cognitive decline (Fig. 5).

\section{Discussion}

We estimated the risk of cognitive decline based on variation pattern of cholesterol different from previous studies. The results showed that increased TC or NHDL-C from normal to high level was significantly associated with decreased risk of global cognitive decline in females compared with stable normal levels. Decreased TC from high to normal level was significantly associated with increased risk of memory function decline both in males and females compared with stable high levels; but only significant in females for decreased NHDL-C. Persistent high levels of TC and NHDL-C did not significantly increase the risk of any cognitive dimensions. For males, decreased LDL-C from high to normal level was significantly associated with decreased risk of mental intactness declined, and persistent high levels of LDL-C was significantly associated with increased risk of mental intactness declined. The variation of HDL-C was not associated with cognitive decline.

TC and NHDL-C are well-established risk factors for cardiovascular diseases, diabetes and stroke. Multicenter study has determined a significant association of baseline NHDL-C with long-term cardiovascular events, including coronary heart disease, unstable angina, coronary death, coronary revascularization and ischemic stroke [17]. Because the cardiovascular health is helpful in keeping normal cognition, the role of cholesterol on cognition should be in line with cardiovascular disease. However, the association between cholesterol and cognitive function has been divergent in recent years. A study using urban community participants from Shanghai, China indicated that higher LDL-C was associated with low risk of dementia [6]. There were also randomized trials indicated that the therapy of lowering cholesterol by statins diminished cognitive function slightly $[18,19]$. Therefore, the effect of cholesterol variability on cognition remains confusing.

Previous studies have been clarified associations of higher visit-to-visit TC, NHDL-C and LDL-C with cognitive decline $[10,12,20]$. In these studies, CV, VIM and SD reflected the degree of dispersion of cholesterol including downward and upward fluctuations. Not only the stability of cholesterol, but also the long-term unidirectional trend was important to cognitive function. But in previous studies, we still don't know which direction of cholesterol variation can protect cognition in long-term observation. Now we found that the variations of TC and NHDL-C from normal to high level had a protective role on cognitive function, which were in inconformity to results from CV, VIM and SD. There was no significant difference in CV, VIM and SD between normal-high and high-normal groups, and the high CV, VIM and SD may also appeared both in normal and abnormal cholesterol range, which led to ambiguity of association between the direction of cholesterol variation and cognitive decline.

Jianian Hua et al. found a negative association between intra-individual SD and memory function in males using the same survey data as our present study [12]. Stable cholesterol is certainly favorable for cognitive ability. But we did found that the association between unidirectional variation of cholesterol and 
cognitive decline was differently from that CV, VIM or SD. Generally, we only carry out lipid-lowering intervention when blood lipids exceed the ideal levels. So our results added certain guiding significance for whether lipid-lowering intervention.

Several studies support our findings. A study based on Finland population indicated that moderate decreased serum TC from midlife to late life was associated with 3.5 times risk of cognitive impairment [21]. The same conclusion was documented in Sweden females, in which the lowest quartile of TC difference (refers to greatest decrease in TC) after 32 years follow up was associated with 2.37 times risk of dementia [22]. In Japanese male population, the decreased trend of serum TC was occurred at least 15 years before the diagnosis of dementia, and was lower than those without dementia [23]. In a birth cohort study from Germany, participants with incidence of mild cognitive impairment and Alzheimer's disease had decreased TC level from baseline to endpoint but remained stable in those who remained healthy [24]. The above studies suggested that TC was protective for cognitive function which was consistent with our study. After subtracted HDL-C from TC, the same results for NHDL-C still existed. LDL-C is the main component of NHDL-C. However, the variation of LDL-C was negatively associated with cognitive function in our study, although only in males. This suggested that thoughtful consideration of selective regulate lipids for improving cognitive function is needed.

The positive association of TC and NHDL-C with cognitive function may be partly explained by BMI. Higher BMI is one consequence of hypercholesterolemia [25]. In our data, we found a lower baseline BMI among participants with onset of cognitive decline in 2015. The lower BMI may likely to induce a decreased tendency of TC or NHDL-C. Another critical factor affects cognitive function is age. But in our analysis, there was no difference in age among four groups of cholesterol. The cognitive tendency was comparable for each group individuals in terms of age. An interesting finding was that the association between LDL-C and cognitive decline was opposite to that between TC, NHDL-C and cognitive decline, although only for mental intactness in males. This may be explained through various lipoprotein transport mechanisms. LDL transports cholesterol to peripheral tissues, which is more sensitive to the accumulation of peripheral inflammation and lipid plaques; while HDL reverses transports cholesterol from peripheral tissues to liver for recirculation or excretion in the form of bile acid [26]. TC contains LDL$\mathrm{C}$ and HDL-C. The respective association of LDL-C and HDL-C with cognition may be obscured by this synthesis due to the opposite effect of members. NHDL-C contains LDL-C, which demonstrated that nonLDL-C and NHDL-C cholesterols were key betrayal of LDL-C.

The menstruation of females may be a cause of gender-specific for association of cholesterol with cognitive decline. Postmenopausal females have less estrogen, which reduced level of cholesterol ester transfer protein [27]. The presence of menopause makes more violent fluctuation of cholesterol in females than that in males. Indeed, we found the number of females was significant higher than male in normal-high, high-normal and high-high group. Even in premenopausal with a regular menstrual cycle, the effect of estrogen is stronger than that androgen in males. We also adjusted menstrual status in subgroup. Meanwhile, the differences in sample size result from gender-distribution should be noticed. Sample size is a factor that cannot be ignored for population study. Furthermore, in normal-high, high- 
normal and high-high group, the age of females was older than normal-normal group, which was more sensitive to cognitive decline; but the age of males was younger. This was consistent with that older females were more likely to be postmenopausal, and had a higher level of TC, NHDL-C or LDL-C. Therefore, the female may bear the brunt of dyslipidemia and cognitive impairment.

\section{Conclusion}

In conclusion, the longitudinal decrease of TC or NHDL-C was associated with subsequent worse cognitive functions among middle-aged and elderly Chinese adults. This association was inversely between LDL-C and cognitive function. The female was more sensitive to cholesterol and cognitive variation. This study adds further evidence that lipids regulation strategies based on gender and the type of cholesterol should be considered in primary prevention of cognitive impairment.

\section{Limitations}

In present study, we precisely clarified the association between variation of cholesterol from high to normal level and cognitive decline, as well as the gender-difference. But there were several limitations. First, we only observed a positive association of longitudinal increased TC and NHDL-C for cognitive function, but the time of cognitive decline was useless because the blood testing was only carried out in 2011 and 2015. We can't derive context between cholesterol and cognitive variation. Second, we excluded most of participants who did not provide blood sample in 2011 or 2015 or who had cognitive impairment in baseline and this may induce selection bias. Our Supplemental Table 1 showed that most characteristics were significant different between excluded and included participants. Third, cognitive decline was a chronic and long-term outcome, while the follow up was only four years in our study, the longer follow up are required in future study. Fourth, there were no standard for decline of memory and mental health; we defined the last tertile of difference in episodic memory or mental intactness score from 2011 to 2015 as significant decline for cognitive dimension, which may lead to mis-judgment. Last, the $\mathrm{OR}$ and $95 \% \mathrm{Cl}$ were in marginal significance for TC and NHDL-C, which may affect the accuracy of the conclusion. Nonetheless, there was still significant trend even though slightly beyond the range of the ideal $\mathrm{Cl}$.

\section{Abbreviations}

BMI, body mass index; CDC, Centers for Disease Control and Prevention; CHARLS, China Health and Retirement Longitudinal Study; $\mathrm{Cl}$, confidence interval; CV, coefficient of variation; HDL-C, high-density lipoprotein cholesterol; LDL-C, low-density lipoprotein cholesterol; NHDL-C, non-high-density lipoprotein cholesterol; OR, odds ratio; SD, standard deviation; TC, total cholesterol; VIM, variability independent of the mean.

\section{Declarations}


Acknowledgements

The development of the Harmonized CHARLS was funded by the National Institute on Aging (R01 AG030153, RC2 AG036619, R03 AG043052). We appreciated the China Center for Economic Research, the National School of Development of Peking University for providing the data.

\section{Authors' contributions}

$\mathrm{HL}$ and XW contributed to the study concept and design; $\mathrm{HL}, \mathrm{RZ}$ conducted the data analysis; $\mathrm{HL}, \mathrm{XW}$ and JZ drafted and critically revised the manuscript; MZ reviewed language and made substantial interpretation of data; $\mathrm{KW}, \mathrm{ZH}$ and $\mathrm{ZY}$ collected data. All authors read and approved the final manuscript.

\section{Funding}

This study was supported by the Natural Science Foundation of Guangdong Province and Science and technology plan of Guangzhou City.

\section{Availability of data and materials}

The CHARLS dataset is freely available to all bona fide researchers. Researchers can gain access to the data (http://charls.pku.edu.cn/en). Data can also be obtained on request (statguo@ccmu.edu.cn).

\section{Ethics approval and consent to participate}

The survey was approved by the Institutional Review Board of Peking University, China (IRB0000105211015). All subjects provided written informed consent in baseline survey and follow up.

\section{Consent for publication}

All authors approved the final manuscript for submission and gave consent for publication.

\section{Competing interests}

The authors declare that they have no competing interests.

\section{References}

1. Pettigrew C, Soldan A, Wang J, et al. Association of midlife vascular risk and AD biomarkers with subsequent cognitive decline. Neurology. 2020. 95(23): e3093-e3103.

2. Stough C, Pipingas A, Camfield D, et al. Increases in total cholesterol and low density lipoprotein associated with decreased cognitive performance in healthy elderly adults. Metab Brain Dis. 2019. 34(2): 477-484.

3. McFarlane O, Kozakiewicz M, Kędziora-Kornatowska K, et al. Blood Lipids and Cognitive Performance of Aging Polish Adults: A Case-Control Study Based on the PolSenior Project. Front 
Aging Neurosci. 2020. 12: 590546.

4. Suemoto CK, Szlejf C, Santos IS, et al. Ideal vascular health and cognitive performance in the Brazilian Longitudinal Study of Adult Health. Eur J Neurol. 2021. 28(1): 71-80.

5. Panza F, Solfrizzi V, D\&\#39, et al. Higher total cholesterol, cognitive decline, and dementia. Neurobiol Aging. 2009. 30(4): 546-8.

6. Zhou F, Deng W, Ding D, et al. High Low-Density Lipoprotein Cholesterol Inversely Relates to Dementia in Community-Dwelling Older Adults: The Shanghai Aging Study. Front Neurol. 2018. 9: 952.

7. Rej S, Saleem M, Herrmann N, Stefatos A, Rau A, Lanctôt KL. Serum low-density lipoprotein levels, statin use, and cognition in patients with coronary artery disease. Neuropsychiatr Dis Treat. 2016. 12: 2913-2920.

8. Giugliano RP, Mach F, Zavitz K, et al. Cognitive Function in a Randomized Trial of Evolocumab. N Engl J Med. 2017. 377(7): 633-643.

9. Ancelin ML, Ripoche E, Dupuy AM, et al. Gender-specific associations between lipids and cognitive decline in the elderly. Eur Neuropsychopharmacol. 2014. 24(7): 1056-66.

10. Smit RA, Trompet S, Sabayan B, et al. Higher Visit-to-Visit Low-Density Lipoprotein Cholesterol Variability Is Associated With Lower Cognitive Performance, Lower Cerebral Blood Flow, and Greater White Matter Hyperintensity Load in Older Subjects. Circulation. 2016. 134(3): 212-21.

11. Lee SH, Han K, Cho H, et al. Variability in metabolic parameters and risk of dementia: a nationwide population-based study. Alzheimers Res Ther. 2018. 10(1): 110.

12. Hua J, Qiao Y, Ke C, Shen Y. Higher visit-to-visit total cholesterol variability is associated with lower cognitive function among middle-aged and elderly Chinese men. Sci Rep. 2020. 10(1): 15555.

13. Adults J. Guidelines for the prevention and treatment of dyslipidemia in Chinese adults (revised in 2016). Chinese Circulation Journal. 2016. 31(10): 937-953.

14. Baars MA, van Boxtel MP, Dijkstra JB, et al. Predictive value of mild cognitive impairment for dementia. The influence of case definition and age. Dement Geriatr Cogn Disord. 2009. 27(2): 17381.

15. Seo EH, Lee DY, Kim SG, et al. Validity of the telephone interview for cognitive status (TICS) and modified TICS (TICSm) for mild cognitive imparment (MCI) and dementia screening. Arch Gerontol Geriatr. 2011. 52(1): e26-30.

16. Crimmins EM, Kim JK, Langa KM, Weir DR. Assessment of cognition using surveys and neuropsychological assessment: the Health and Retirement Study and the Aging, Demographics, and Memory Study. J Gerontol B Psychol Sci Soc Sci. 2011. 66 Suppl 1(Suppl 1): i162-71.

17. Brunner FJ, Waldeyer C, Ojeda F, et al. Application of non-HDL cholesterol for population-based cardiovascular risk stratification: results from the Multinational Cardiovascular Risk Consortium. Lancet. 2019. 394(10215): 2173-2183. 
18. Muldoon MF, Ryan CM, Sereika SM, Flory JD, Manuck SB. Randomized trial of the effects of simvastatin on cognitive functioning in hypercholesterolemic adults. Am J Med. 2004. 117(11): 8239.

19. Ying H, Wang J, Shen Z, Wang M, Zhou B. Impact of Lowering Low-Density Lipoprotein Cholesterol with Contemporary Lipid-Lowering Medicines on Cognitive Function: A Systematic Review and MetaAnalysis. Cardiovasc Drugs Ther. 2020 .

20. Grasset L, Smit R, Caunca MR, et al. Association of High-Density Lipoprotein Cholesterol With Cognitive Function: Findings From the PROspective Study of Pravastatin in the Elderly at Risk. J Aging Health. 2020. 32(9): 1267-1274.

21. Solomon A, Kåreholt I, Ngandu T, et al. Serum cholesterol changes after midlife and late-life cognition: twenty-one-year follow-up study. Neurology. 2007. 68(10): 751-6.

22. Mielke MM, Zandi PP, Shao $\mathrm{H}$, et al. The 32-year relationship between cholesterol and dementia from midlife to late life. Neurology. 2010. 75(21): 1888-95.

23. Stewart R, White LR, Xue QL, Launer LJ. Twenty-six-year change in total cholesterol levels and incident dementia: the Honolulu-Asia Aging Study. Arch Neurol. 2007. 64(1): 103-7.

24. Toro P, Ch D, Pierer M, Gustafson D, Schröder J, Schönknecht P. Cholesterol in mild cognitive impairment and Alzheimer's disease in a birth cohort over 14 years. Eur Arch Psychiatry Clin Neurosci. 2014. 264(6): 485-92.

25. Lu Q, Guo P, Liu A, et al. The role of long noncoding RNA in lipid, cholesterol, and glucose metabolism and treatment of obesity syndrome. Med Res Rev. 2020 .

26. Duran EK, Aday AW, Cook NR, Buring JE, Ridker PM, Pradhan AD. Triglyceride-Rich Lipoprotein Cholesterol, Small Dense LDL Cholesterol, and Incident Cardiovascular Disease. J Am Coll Cardiol. 2020. 75(17): 2122-2135.

27. Guo Y, Zhao M, Bo T, et al. Blocking FSH inhibits hepatic cholesterol biosynthesis and reduces serum cholesterol. Cell Res. 2019. 29(2): 151-166.

\section{Figures}



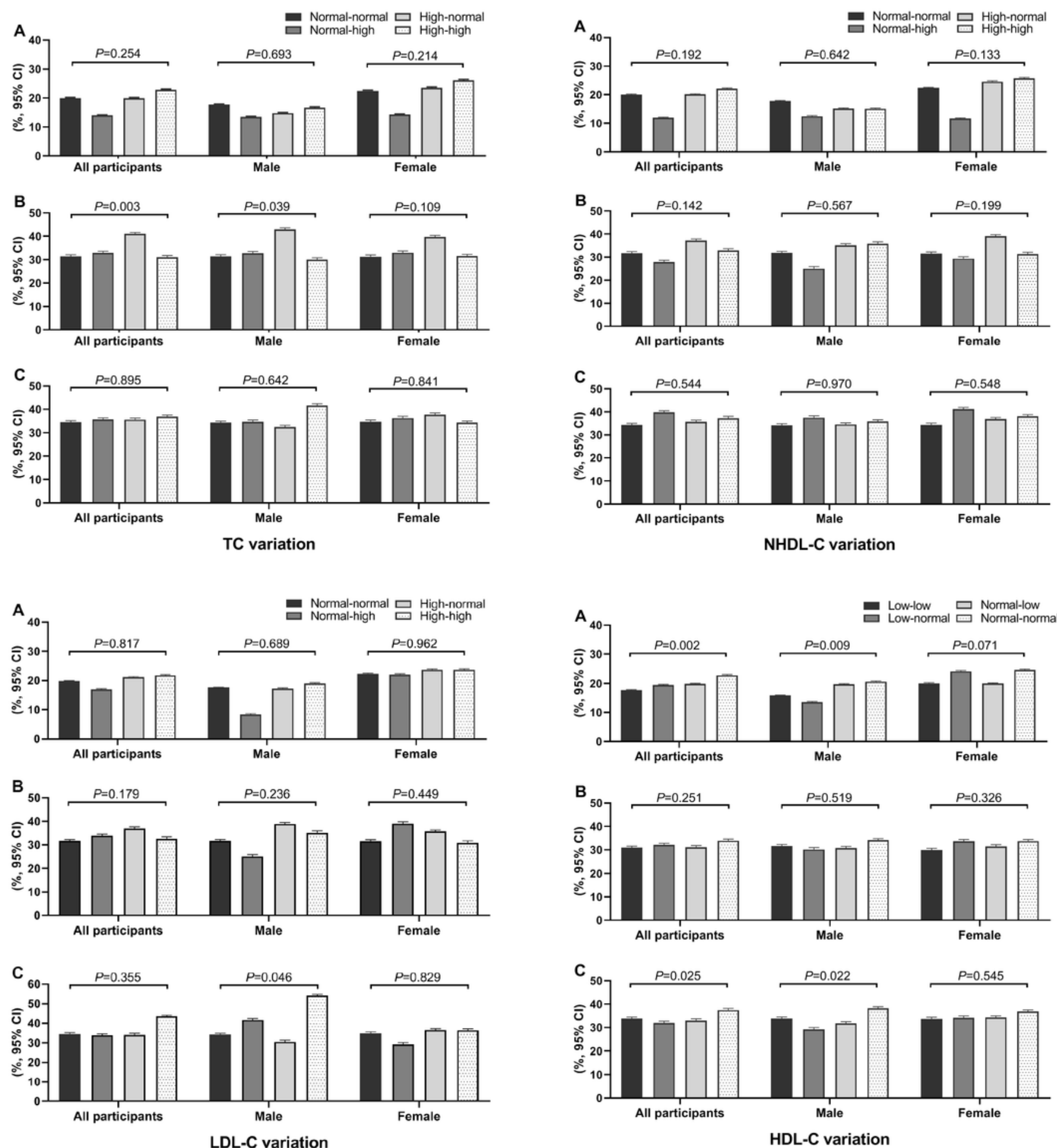

HDL-C variation

\section{Figure 1}

The incidence rate of cognitive decline among cholesterol groups. A, global cognitive decline; B, Memory function decline; C, Mental intactness decline; TC, total cholesterol; NHDL-C, non-high-density lipoprotein cholesterol; LDL-C, low-density lipoprotein cholesterol; HDL-C, high-density lipoprotein cholesterol. 


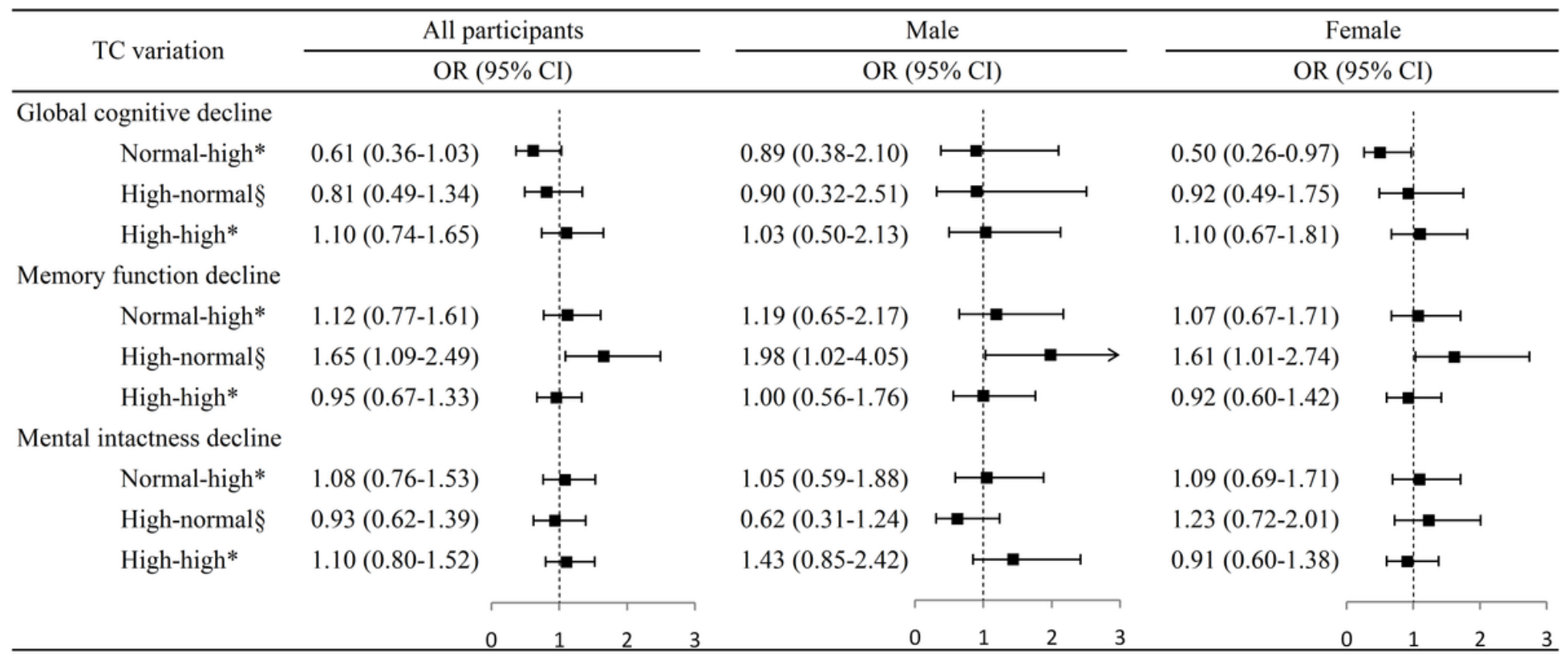

\section{Figure 2}

Association between TC variation and cognitive decline. TC, total cholesterol; OR, odds ratio; $\mathrm{Cl}$, confidence interval. *The normal-normal group was reference; §The high-high group was reference. Baseline age, sex, education, marital status, smoking, drinking, BMl, physical diseases and two years average cholesterol level were adjusted.

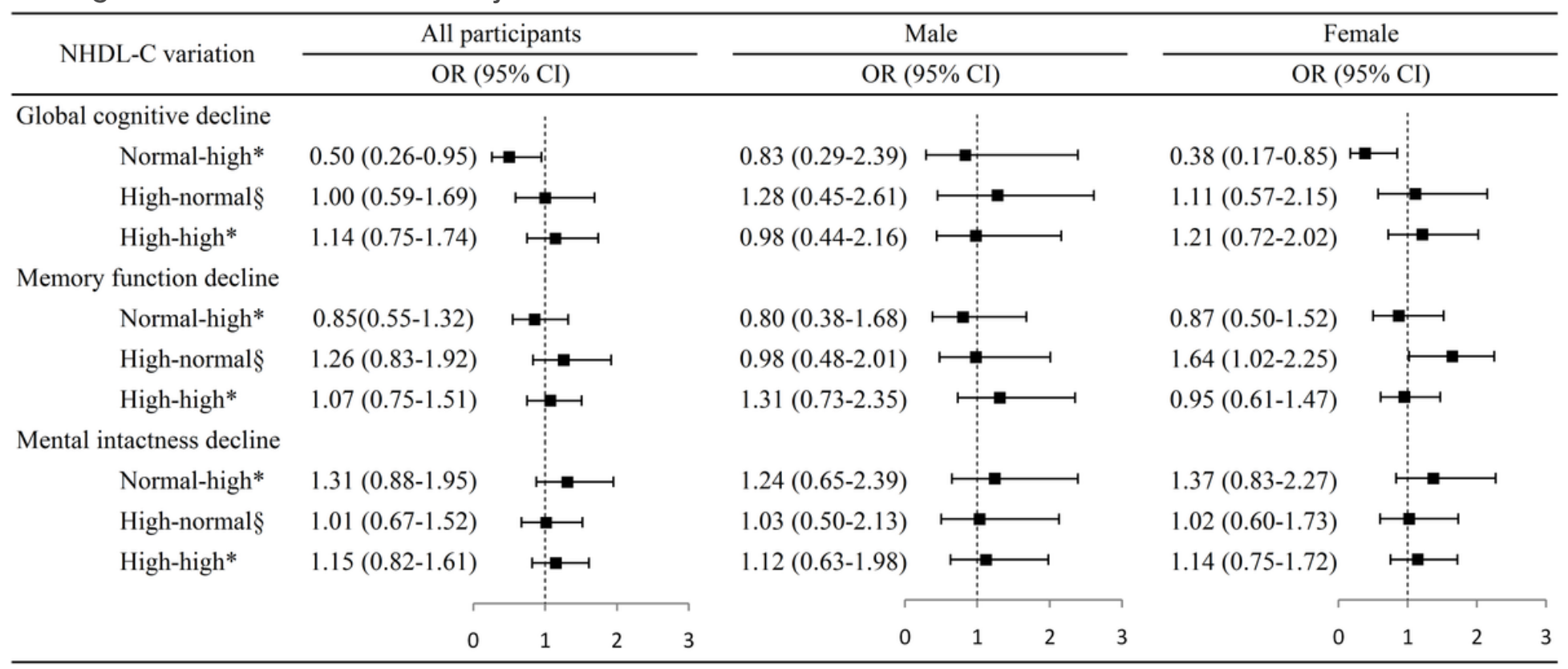

\section{Figure 3}

Association between NHDL-C variation and cognitive decline. NHDL-C, non-high-density lipoprotein cholesterol; OR, odds ratio; $\mathrm{Cl}$, confidence interval. *The normal-normal group was reference; §The highhigh group was reference. Baseline age, sex, education, marital status, smoking, drinking, BMI, physical diseases and two years average cholesterol level were adjusted. 


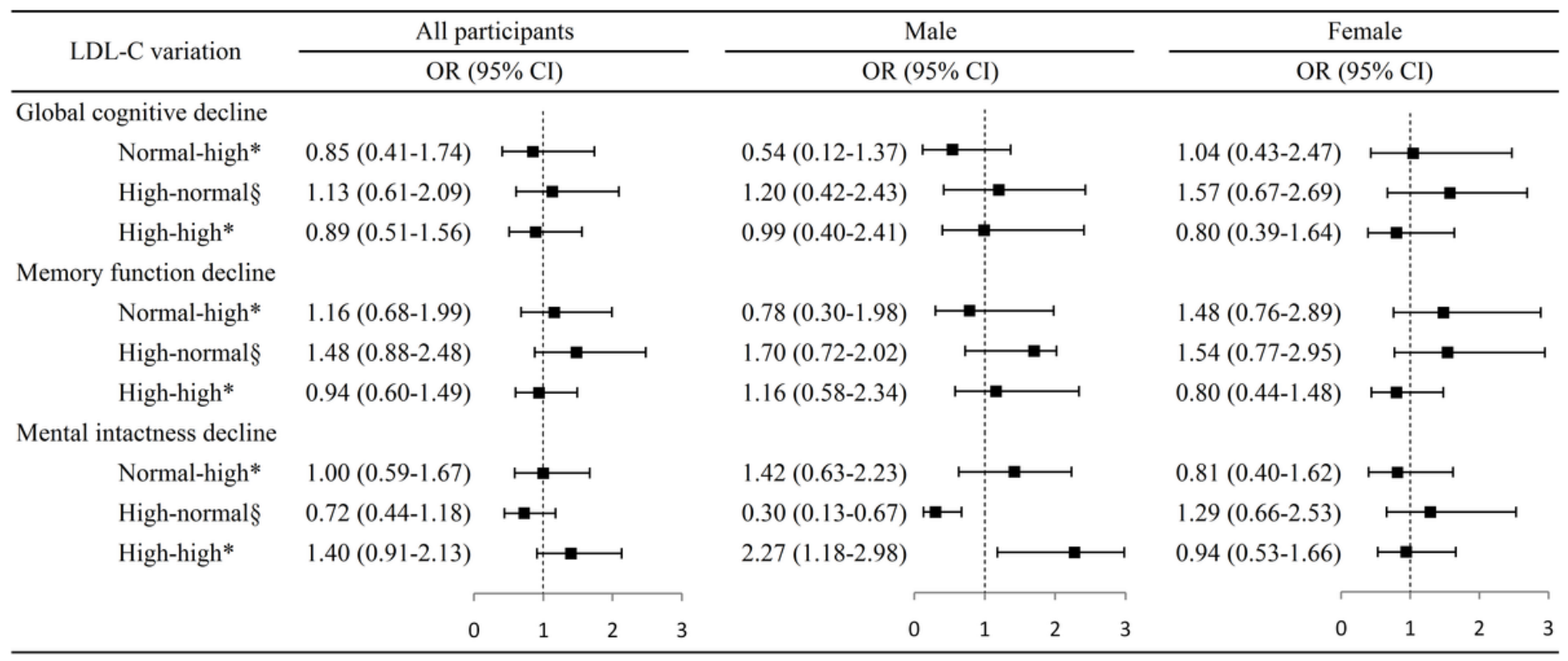

\section{Figure 4}

Association between LDL-C variation and cognitive decline. LDL-C, low-density lipoprotein cholesterol; OR, odds ratio; $\mathrm{Cl}$, confidence interval. *The normal-normal group was reference; §The high-high group was reference. Baseline age, sex, education, marital status, smoking, drinking, BMI, physical diseases and two years average cholesterol level were adjusted.

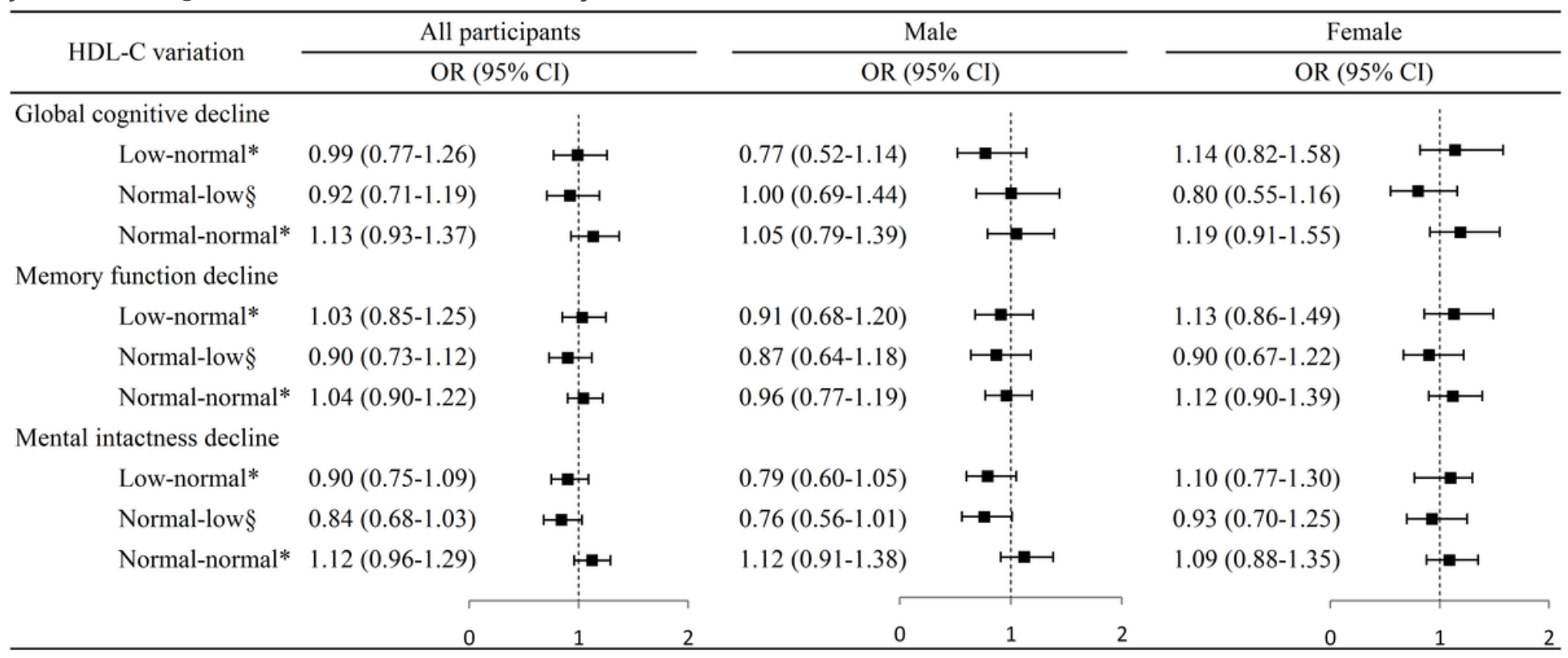

\section{Figure 5}

Association between HDL-C variation and cognitive decline. HDL-C, high-density lipoprotein cholesterol; $\mathrm{OR}$, odds ratio; $\mathrm{Cl}$, confidence interval. *The normal-normal group was reference; §The high-high group was reference. Baseline age, sex, education, marital status, smoking, drinking, BMI, physical diseases and two years average cholesterol level were adjusted. 


\section{Supplementary Files}

This is a list of supplementary files associated with this preprint. Click to download.

- SupplementalFigure1Flowchart.tif

- SupplementalTables.docx 